\title{
Conferencia
}

\section{A 150 años del origen de las especies}

\author{
JAIME MAÑALICH. MINISTRO DE SALUD. CHILE
}

Muy buenos días a todos, mucho gusto de verlos, sobre todo a tanta gente joven. Es esperanzador respecto al quehacer de las políticas públicas de salud en nuestro país. Esta presentación se basa en que a principios del siglo XXI tenemos una mirada clara respecto a la filosofía política que debe animar la definición de políticas sanitarias para nuestro país, y como país inserto en el mundo que es. Las tensiones que existen en esta materia son muchas y he tomado una posición muy discutible, pero de eso se trata también, que es tratar de responder a la pregunta respecto a si es coincidencia, a 150 años de publicación del libro, en mi opinión, más importante de la humanidad después de El antiguo testamento, como es El origen de las especies, que acaso hay en esta celebración un paradigma, una forma de ver las cosas que determine o defina políticas sanitarias. Esto, evidentemente, no es nuevo. Cuando Bismark en el año 1881 hace sus tres leyes de beneficio social, de inserción laboral y de protección de la salud, hay una visión filosófica y antropológica respecto a qué es el hombre, cuál es el rol del ser humano en el Universo, qué es lo que ha enseñado Kant respecto al imperativo ético; y todo eso se traduce en políticas públicas.

En mi opinión, el desafío más grande que enfrenta la humanidad a inicios del siglo XXI, es que es un ensayo biológico, una creación -como ustedes lo quieran ver-, bastante nueva en la historia de nuestros 4 mil millones de años de planeta Tierra. Tenemos el conocimiento de 150 mil años de antigüedad aproximadamente y somos un modelo diseñado para vivir en grupos de 40, 50 personas; para comer determinadas cosas, raramente carne, muchas veces pescado crudo; en sociedades colectivas muy matriarcales. Y esto se ha mantenido así prácti- camente, insisto, por los últimos 150 mil años.

Los cambios o desafíos a este modelo o imperativo biológico son muy recientes y están representados en que, habiendo sido un modelo biológico para una expectativa de vida de más o menos 30 a 35 años, vale decir, reproducirse y listo, en los poquísimos recientes años hemos hecho algo increíble sólo con instrumentos, al final, culturales: hemos sido capaces de extender la expectativa de vida a aproximadamente 80 años. Esto es una cosa absolutamente nueva, nunca antes en la historia hubo un momento en el cual una cultura, una civilización, se haya enfrentado a un desafío de esta magnitud, y lo tenemos que decir claramente: no tenemos la más remota idea de cómo resolverlo.

En Chile, como país, tenemos una ventana que llamamos un bono demográfico y que dura muy poquito, más o menos 30,35 años, en los cuales vamos a tener algunas generaciones de gente más sana, más vigorosa, mejor educada, que se va a incorporar al mundo de la educación, del trabajo o lo que fuere, y también al mundo de la reproducción. Todo ello, antes de que lleguemos a ser un país viejo y pobre. Y cómo definamos las políticas públicas durante estos años, significa un movimiento de ángulo muy pequeño, que nos lleva allá o acá. En ese sentido, el desafío que tenemos como hijos de este tiempo, es -insisto- completamente nuevo. Y a diferencia de lo que ocurría con la medicina social de los años 1920 a 1930 en Chile, cuando básicamente se trataba de adaptar a nuestro país políticas que estaban siendo exitosas, sobre todo en Europa, no tenemos hoy un modelo claro al cual mirar. Este desafío se podría haber resuelto de una manera simple y es haber invocado al Creador para que hiciera un ser humano 2.0 con algunos cambios; pero 
hasta la fecha no se sabe que esto haya ocurrido como solución al desafío que enfrentamos.

En muy pocos años, nos encontramos con otro problema: hoy, la medicina curativa sirve. Hoy la Medicina es eficaz, a gran costo, pero es eficaz, pues produce la curación de las enfermedades y esta tremenda revolución, si uno la mira en la perspectiva del tiempo, es muy reciente y de todos los días, en que se encuentran procedimientos que producen mayoritariamente sanación de personas que están enfermas de algo. Y eso, insisto, cuando se soñaba como un acceso universal a la salud el año 2000, no estaba en la ecuación. No sabíamos que íbamos a tener una explosión tecnológica de medicina curativa de tal magnitud que cambiaría completamente nuestra aproximación a la enfermedad. Cuando yo estudié Medicina, las leucemias de los niños eran enfermedades mortales, pero hoy si un niño se muere de una leucemia es una cosa rarísima. Y, efectivamente, nuestro país, al igual que todo el mundo, está gastando cada vez más recursos en curar a las personas y, enfatizo, en curar a las personas una vez que están enfermas. Nos gastamos una cantidad importante de recursos públicos en eso.

Aquí está el incremento real en gasto en salud en Chile durante la última década, del orden del $10 \%$, independiente de cómo haya sido la economía en el país, incluso en momentos en que, por ejemplo en 2009, decreció violentamente, nosotros seguimos incrementando el gasto en salud por esta disyuntiva. El presupuesto del año 2011, recién aprobado, es de nuevo inflacionario. Subimos un $13 \%$, un aumento sideral para una expansión del gasto público de un total de $5 \%$, pasando a una cifra de más o menos U\$8 mil millones, en circunstancias que en moneda del mismo valor el año pasado había sido de U\$6.400 millones. Llegamos a una situación en que por primera vez el porcentaje del gasto en salud pública como representación de una parte del gasto fiscal, llega a ser el $15 \%$, en circunstancias que siempre había sido inferior a dos dígitos. No es que no nos hagamos cargo del problema, sino que estamos gastando cada vez más recursos.

Aquí tenemos que hacer un paréntesis. En el Iluminismo del siglo XIX, entender qué era el hombre era una dificultad enorme; no digamos que hoy es más fácil, pero en ese tiempo era un problema gigantesco. Y así, la interpretación esencial era la escritura, el Génesis, capítulo 1, donde se decía que Dios creó las cosas y al hombre, por supuesto, de una manera que todos conocemos. Dicen que si se traduce la expresión "be-reshit", que es la expresión hebrea del Principio, la traducción exacta es que Dios empezó a crear el mundo como un proceso creativo continuo. Esta forma de ver las cosas llevó al Arzobispo James Ussher a calcular cuándo fue creada la Tierra, de acuerdo al relato bíblico, vale decir, la fecha exacta es aproximadamente 6 mil años atrás, que en realidad es la historia consciente de la humanidad. Sin embargo, las Ciencias buscaban mecanismos, detrás de la mecánica, que era el paradigma esencial con el cual tratar de entender las cosas y cómo funcionan. Y, evidentemente, estos dos personajes son esenciales en señalar que el Universo tiene reglas prácticamente inamovibles, por las cuales actúa y se mueve, y las cosas tienen su horizonte de posibilidades. Carlos Linneo sugiere que algo parecido puede ocurrir para los seres vivos y el primer intento que hace es de clasificarlos, lo que de alguna manera da un pie inmediato para lo que viene a continuación. En el intento de conciliación entre los datos geológicos, los datos biológicos, el hallazgo de los primeros fósiles en Francia de especies que ya no existían, huesos que eran de especies ya desaparecidas, aparece este clérigo británico y dice: en realidad, hay un Dios creador que se manifiesta progresivamente en su quehacer a lo largo de la historia y uno puede hacer una Teología Natural, de manera tal de descubrir -en estilo de Aristóteles o Santo Tomás de Aquino- dónde están las huellas de esa creación en el mundo real, en el mundo de los seres vivos. Quien da un paso adelante en esto es Lamarck. Este sabio francés dice que en realidad las especies cambian, evolucionan, y propone un mecanismo y es que en la medida en que determina- 
das condiciones cambian a lo largo de la vida de un individuo biológico, estas adaptaciones que son para mejor, son capaces de ser transmitidas por un mecanismo desconocido a su descendencia. Y da el ejemplo de la jirafa, a la que le crece el cuello a medida que busca las hojas que están más altas en los árboles.

Un personaje extraordinariamente interesante es Erasmus Darwin, abuelo de Charles. Este hombre escribe su libro "Zoonomia" a fines del siglo XVIII, en el cual dice: “¿Cuáles son las leyes de la vida orgánica?”. Y es primera vez en la historia de las ciencias humanas que alguien habla de leyes naturales, es decir, leyes inmanentes al ser biológico, que gobiernan -al igual como lo habían hablado Copérnico y Newton en relación a la Esfera Celeste- la vida orgánica. Este señor tuvo un nieto catalogado de "bueno para nada", Charles, cuyo padre desesperado decidió embarcarlo en un largo periplo por el mundo para ver si sentaba cabeza y llegaba a ser alguien. El viaje del Beagle duró entre 1931 y 1936 , pero lo interesante fue que gracias a él Charles Darwin estuvo en Chile desde diciembre de 1932 hasta julio de 1935, y si uno lee los apuntes de Darwin y recorre lo que él anotaba mientras estaba acá, partió todo en Chile, siendo bien chovinista. Y esto ocurre porque mientras él recala, recorre, viaja a Los Andes, se hace preguntas en su cuaderno que son fascinantes. Por ejemplo: “¿Por qué el Divino Hacedor se dio la molestia de crear desde el principio un roedor de un tipo acá y uno del otro tipo, allá?". Estos cuestionamientos maduran en las islas Galápagos, que Darwin recorre y se da cuenta que en aquellas donde la vegetación es más rara, las tortugas son capaces de levantarse en sus patas y levantar el cuello, y están hechas para eso; en cambio, donde todo es más cómodo para ellas, las mismas son completamente diferentes. Y de nuevo Darwin se pregunta por qué es así.

El 20 de febrero de 1835 se produce un tremendo terremoto en Concepción. Darwin está en las afueras de Valdivia y luego logra recalar en Talcahuano. Inmediatamente él recorre el lugar y se pregunta por qué la tierra se ha levantado de esta manera. Uno va a Lebu hoy después del terremoto de febrero de este año y la ría de Lebu, donde se pescaba antes del sismo, no existe, y cambiaron las especies; o sea, son cosas que ocurren todos los días, pero Darwin fue capaz de mirarlas y hacerse preguntas cruciales. Ya retirado, Charles Darwin tuvo la oportunidad de conocer dos textos esenciales para la conformación de su modelo. El más importante fue "El tratado sobre la población humana" de Robert Malthus, padre de la demografía, en el cual este autor señala que mientras las especies crecen en proporción geométrica, la posibilidad de producir alimentos lo hace en proporción aritmética. Entonces, ese desbalance genera una presión por la vida de tal magnitud que lleva a la selección de las especies.

Así, Darwin descubre que el mecanismo por el cual funciona este modelo es que la naturaleza es la gran seleccionadora. Y cuando llega a su Inglaterra natal, elabora lo que denomina "el árbol de la vida" y señala lo que él cree, una cuestión de una genialidad pocas veces vista en la esquina de un cuaderno. Darwin se da cuenta de la potencia de su teoría y decide no decir nada. Sin embargo, aparece alguien, Wallace, quien llega a la expresión de una teoría similar a la de Darwin $\mathrm{y}$ en un gesto impresionante y reconociendo la autoridad moral de Darwin, se lo manda para revisión. De esta forma, el 30 de junio de 1858 donde se presentan dos papers, uno de Wallace y otro de Darwin, con lo que en definitiva se da origen a la teoría de la evolución. Pero Darwin la tenía tan elaborada que al año siguiente, en 1859, publica su libro que se llama "El origen de las especies por medio de la selección natural" y con el subtítulo: "La preservación de las razas más favorecidas en la lucha por la vida".

A estas alturas, Darwin ya había recibido la influencia de Herbert Spencer, el padre de la Sociología, quien fue el real acuñador de la expresión de la supervivencia de los más fuertes. Y dan origen él y el sobrino de Darwin, sir Francis Galton, a todo lo que conocemos hoy como evolucionismo social, que tuvo pro- 
bablemente en la primera mitad del siglo XX su expresión más tremenda, más desastrosa y horrorosa para el recuerdo de las generaciones que nos sucederán. Nosotros hablamos de la Edad Media como de los años oscuros, pero yo les aseguro que cuando en el siglo XXIII se mire la primera mitad del siglo XX, la Edad Media va a ser la primavera al lado de lo que fue la primera mitad del siglo XX.

Desde el punto de vista de la Biología es interesante ver que este modelo ha sido aplicado cada vez más sistemáticamente al hombre y a sus enfermedades, a la forma en que es concebida la evolución de algunos órganos. Por ejemplo, el ojo, las articulaciones, etcétera. Y esto se entronca con los descubrimientos de Mendel que fueron simultáneos a los de Darwin, a pesar de que no se conocieron. Esto da origen a lo que se conoce como el Modelo Neodarwinista, en el cual se produce la síntesis entre los modelos descritos por Darwin y el mecanismo descrito por Mendel, lo que culmina cuando Watson y Crick dicen: esta es la razón de fondo. Hoy el lenguaje evolutivo está en todas partes. Es impresionante cómo cualquier niño conoce de qué forma cambian las especies, a tal punto que nos encontramos incluso en el cine con expresiones evidentemente sensatas relativas a la teoría de la evolución. A nadie le cabe duda que esta discusión está ya zanjada.

En Biología, nada hace sentido si no se interpreta a la luz de la evolución. Y yo lo voy a decir esta mañana: nada en salud pública tiene sentido si no se mira a la luz del marco evolutivo; todo lo otro es perder el tiempo. Nada se puede mirar en enfermedad, en medicina, en salud pública si no es de esa forma y los problemas que produce la evolución. Y así, para la Medicina Clínica hoy día la rama de desarrollo evolutivista es un dato, o sea, todo el mundo está empezando a pensar: ¿Por qué nos enfermamos? La nueva ciencia de la medicina darwiniana: Medicina Evolutiva. Y el modelo esencial es que el origen de la enfermedad en estos siglos se debe esencialmente a lo que yo denomino como una brecha evolutiva, es decir, una profunda desadaptación de lo que so- mos como modelo biológico y las condiciones en las que estamos viviendo en el siglo XXI. La mayor parte del quehacer en salud pública tiene que ver con esta pregunta: ¿De qué magnitud es el desbalance? ¿De qué magnitud es la brecha y cómo nos arreglamos para superarla? Porque después de la experiencia de inicios del siglo $\mathrm{XX}$, la humanidad ha tomado una decisión ética y moral que es comportarse de manera antievolutiva. O sea, nosotros no aceptamos que los más débiles, los más enfermos sean seleccionados negativamente, sino que hemos tomado la opción moral de decir: no es así. Y vamos a involucrar todos nuestros esfuerzos por vivir y por superar esa brecha evolutiva.

El crecimiento de la próstata después de los 50 años, la depresión, la obesidad, muchas enfermedades se deben a coevolución. Encontramos una bala mágica para matar los antibióticos, el germen evoluciona y se hace resistente; encontramos una cura a la malaria, el cólera... para qué hablar. En la evolución y en la coevolución hay demasiado de la explicación y hay prescripciones, en cuanto a dejar a los parásitos tranquilos para permanecer en equilibrio. Es una experiencia de duro aprendizaje.

Durante la década pasada, en realidad inspirado en la década antepasada, Chile se involucró en una tremenda reforma de salud, que puede ocurrir solo cada 30 años. La perspectiva reformadora -yo no comparto esta visiónes que las atenciones de salud uno las puede dividir en bienes de apropiación privada, vale decir, aquellos bienes que solo tienen que ver conmigo, por ejemplo, la cirugía plástica. Pero otros, como el tratamiento dental, ¿son realmente un bien privado? Algo de lo que tengo que hacerme responsable y si no tengo beneficios o ayuda económica, no puedo. Y así con otras condiciones de salud. En el modelo de prestaciones de acceso universal garantizado, Auge, en el sentido de priorizar algunas condiciones de salud -hoy vamos en $69-$-, el $80 \%$ de los egresos hospitalarios tienen un componente de apropiación pública, porque la persona vuelve al trabajo antes y el sistema de salud pública gasta menos. 
Cuando el Presidente Lagos prescribe la reforma Auge, tomémoslo como paradigma, es evidente que está haciendo una receta magistral bastante importante y hay que reconocer que como toda receta, ésta puede tener efectos adversos que nosotros debemos resolver. Ese es el trabajo: cómo perfeccionamos la reforma Auge en la línea de lo que hemos estado hablando y cuáles son sus efectos positivos y adversos. Yo lo diría en esta frase: nosotros como país le estamos ganando la batalla a la enfermedad. Aquella persona que está enferma tiene más posibilidades hoy día, gracias al Auge, de sanar, de volver a una vida normal y pagar muy poco o pagar de acuerdo a sus ingresos por ese tratamiento. Y ahí está lo que ha ocurrido respecto a la mortalidad por enfermedades isquémicas de acuerdo a la evolución del 2008: se ha reducido notablemente, tenemos un éxito completo.

Pero como efectos adversos a este enfoque ha habido una restricción de los recursos; siempre hubo un paquete preventivo en el Auge que desgraciadamente no hemos podido implementar bien a la fecha. Pero miren lo que está pasando en Chile, somos el segundo país donde el número de suicidios, sobre todo en la gente joven, está aumentando más rápidamente. Es un problema. Miren lo que pasa con la obesidad en escolares de primer año básico, lo que entre 10 y 15 años más significará una carga insostenible para el sistema de salud. Y la forma en que estructuramos nuestra pelea, por así decirlo, por la salud, por ser antievolutivos en la década pasada, fue a través de este documento del año 2002 de Objetivos Sanitarios, en el que básicamente se definen prioridades y se dice por aquí vamos a ir. En la página web del Ministerio de Salud es posible encontrar el documento de evaluación de los objetivos sanitarios de la década que acaba de terminar; y hay una propuesta para vuestra discusión, de cuáles deberían ser los objetivos sanitarios para la década 2011-2020 ,y necesitamos ayuda. Sobre todo en la perspectiva de completar la gran reforma del año 2004-2005. Si uno mira para atrás encuentra cosas interesantes. Uno de los argumentos esenciales para llevar adelante la reforma de salud de la década pasada, Auge, este fue el argumento más repetido: la paradoja Vitacura-Puerto Saavedra. En promedio, como ustedes saben, nosotros tenemos como indicador de la desigualdad en la mortalidad infantil, un cuociente de 5 veces entre las familias más favorecidas, más ricas, y la gente más pobre. Entonces, tenemos que ejercer una acción sanitaria para superar estas brechas. Pero es esto algo que se tiene que hacer desde el punto de vista de la clínica, o la pregunta subyacente es mucho más profunda. Y si ustedes ven, el determinante fundamental de la diferencia de mortalidad en estas comunas no se debe al cuidado hospitalario, sino que el problema se produce en la mortalidad neonatal tardía. El niño llevado por la madre en Puerto Saavedra a su casa, tiene dramáticamente más posibilidades de morir a la semana siguiente, que el niño que se lleva la señora de Vitacura, y eso tiene que ver con el nivel educacional de la madre. Entonces, ¿qué es lo que tenemos que hacer? ¿O tenemos que diseñar un programa para acompañar a estas madres, suponiendo que la equidad económica por el ingreso va a mejorar? ¿Eso es lo que debemos hacer? Son preguntas extraordinariamente relevantes para lograr equidad mediante salud pública.

Por ciento, la forma de crianza hoy día es muy diferente y esto tiene sus consecuencias, a pesar de que hoy es evidente que mantener el contacto de olor (del hijo/a) con la madre es esencial para que los niños no tengan cólicos en la noche, para que se críen más sanos y esto tiene que ver con la ley de posnatal. Y de nuevo estamos frente a una política de salud pública, es decir, de la manera en que se legisle el posnatal, nosotros estamos haciendo una gran política de salud pública hacia el futuro.

Para terminar, yo creo que nuestro desafío es gigantesco y que efectivamente las reformas que ha emprendido nuestro país en los últimos años han sobretecnificado y sobremecanizado el quehacer de la Medicina. Tenemos que volver a la figura del esfuerzo sanitario público como bien de apropiación de todos y para eso necesitamos propuestas concretas. Para eso 
necesitamos ayuda. Y necesitamos sugerencias de políticas que sean efectivas en el sentido de que conduzcan a resultados que probadamente tienen el destino que queremos darles. De manera tal que volviendo a las palabras iniciales, me alegra enormemente ver tanta gente joven aquí, porque de alguna manera tenemos que mover el péndulo, tomar opciones. Y tene- mos dos opciones: o hacemos que todo obeso en Chile se opere de cirugía variática como el paradigma de la salud pública chilena, o reducimos dramáticamente el número de obesos candidatos a cirugía variática en los próximos años. Y esa decisión, con implicancias económicas, sociales y de toda magnitud, en gran medida depende de ustedes. Muchas gracias. 